\title{
Leseliste Top 5: Controlling in China
}

In diesem Teil möchten wir Ihnen regelmäßig fünf besonders lesenswerte Bücher zu einem bestimmten Themengebiet vorstellen:

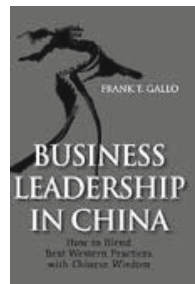

Gallo, Frank T.:

Business Leadership in China

How to Blend Best

Western Practices with

Chinese Wisdom

John Wiley \& Sons, Singapur 2008, 244 Seiten, ISBN-13: 978-0-470-82365-1, Preis: $15,90 €$

Auf der Grundlage seiner reichen Erfahrungen in China geht der Autor zunächst detailliert auf Aspekte der Führung in China ein. Daneben widmet er sich Bereichen, die besonders von soziokulturellen Unterschieden berührt werden, nämlich Teamwork, Entscheidungsfindung und Vertrauen.

Tipp: Das Buch richtet sich an Praktiker in Führungsverantwortung, die mit Mitarbeitern aus dem chinesischen Kulturkreis zusammenarbeiten und verstehen möchten, wie Techniken von Leadership und Management mit Aspekten der chinesischen Kultur fruchtbar kombiniert werden können.

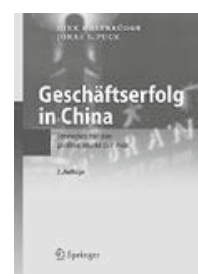

Holtbrügge, Dirk/

Puck, Jonas F.:

Geschäftserfolg in China

Strategien für den größten Markt der Welt

2., überarb. u. erw. Aufl., Springer Verlag, Berlin/Heidelberg 2008, $341 \mathrm{~S}$., ISBN: 978-3-540-79431-8, Preis: 49,95€

Obwohl kaum ein Tag vergeht, an dem die dynamische Wirtschaftsentwicklung in China in den Medien thematisiert wird, muss ein wirtschaftliches Engagement in diesem Land sorgfältig geprüft werden. Leider sind Patentverletzungen, Managementkonflikte oder Probleme mit lokalen Zulieferern immer noch an der Tagesordnung. Hier knüpft das Buch an und liefert an Hand zahlreicher Praxisbeispiele Ent- scheidungshilfen für Unternehmen bezüglich Markteintritt, Marktbearbeitung oder Personalmanagement.

Tipp: Neben zahlreichen Fallstudien bietet das Buch zur Kontaktaufnahme für Praktiker einen umfangreichen Adressteil.

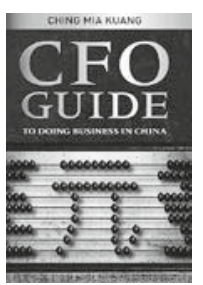

Ching, Mia Kuang:

\section{CFO Guide}

to Doing Business

in China

John Wiley \& Sons, Singapur 2008, 216 S., ISBN: 978-0-470-82373-6, Preis: $24,90 €$

Nicht nur in kultureller, sondern auch in regulatorischer Hinsicht ist China von Unterschieden zu den Staaten der westlichen Welt geprägt. Genau hier setzt der CFOGuide an, der zunächst das Rechnungslegungssystem sowie aktuelle Entwicklungen im chinesischen Steuerrecht betrachtet. Daneben sind Unternehmenskontrollen, Formalitäten bei der Personaleinstellung oder M\&A Thema. Abgerundet wird das Ganze mit Aspekten zur chinesischen Kultur und zu Verhandlungstechniken.

Tipp: Hauptzielgruppe sind Finance-Verantwortliche im China-Geschäft. Aber auch interessierten Praktikern, die über den Bereich soziokultureller Unterschiede hinaus mehr über China erfahren wollen, kann die Lektüre empfohlen werden.

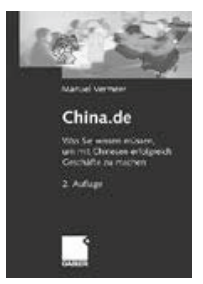

Vermeer, Manuel: China.de

Was Sie wissen müssen, um mit Chinesen erfolgreich Geschäfte zu machen

2., überarb. Aufl., Gabler Verlag, Wiesbaden 2007, 192 S., ISBN: 978-3-8349-0566-6, Preis: 39,90€

Wer die chinesische Kultur verstehen möchte, sollte auch die Historie kennen. Genau damit beginnt der Autor seinen Über- blick über Wesensart und Leben im „Reich der Mitte“. Dies ergänzt er mit Fremdbildern, also der Wahrnehmung der westlichen Nationen bei den Chinesen. Neben Aspekten der Landeskunde und der Kultur gibt Manuel Vermeer wertvolle Hinweise zu Verhandlungsführung und zeigt Fallstricke, die beim Chinaaufenthalt lauern.

Tipp: Das Buch ist für jeden geeignet, den sein beruflicher Weg nach China führt. Dabei ist dieses Handbuch nicht nur für Praktiker, sondern auch für Wissenschaftler hilfreich und interessant, wenn sie in China zu tun haben.

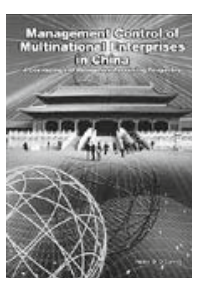

O'Connor, Neale G.:

Management Control of Multinational Enterprises in China:

A Contracting and Management Accounting Perspective

McGraw Hill, New York City 2006, 296 S., ISBN: 978-0-0712-4738-2, Preis: 41,99€

Das Buch befasst sich intensiv mit verschiedenen Aspekten des Management Control in China. Angefangen von der Kontaktherstellung für Investitionen, über weitere Expansionen und das Controlling in Joint Venture bis hin zu Transfer Pricing werden verschiedenste Themen angesprochen. Die fallstudienbasierte Abhandlung der Inhalte verleiht dem Buch viel praktische Authentizität.

Tipp: Zwar richtet sich das Buch eher an Praktiker, aber die eine oder andere Fallstudie kann auch in der Lehre Anwendung finden.

Anton Preis und Julia Schmitz, Vallendar 